\title{
Unidirectional Valved Patch Closure of Ventricular Septal Defect with Eisenmenger Physiology
}

${ }^{1}$ Pankaj Kumar, ${ }^{2}$ Suruchi Hasija, ${ }^{3}$ Usha Kiran

\begin{abstract}
Pulmonary hypertension in left-to-right shunt lesions, such as ventricular septal defect (VSD), patent ductus arteriosus, atrial septal defect (ASD) and atrioventricular canal defect is termed hyperkinetic pulmonary hypertension. Hyperkinetic pulmonary hypertension is usually reversible if the cause is treated before development of permanent changes in pulmonary arterioles. A unidirectional flap valve (UVP) VSD patch closure is a good alternative in children with large VSD with Eisenmenger physiology. The technique of unidirectional valved patch VSD closure obviates the need for extracorporeal membrane oxygenation and nitric oxide ventilation in patients who are at risk of pulmonary hypertensive crises postoperatively.
\end{abstract}

Keywords: Eisenmenger physiology, Pulmonary hypertension, Unidirectional valved patch, Ventricular septal defect.

How to cite this article: Kumar P, Hasija S, Kiran U. Unidirectional Valved Patch Closure of Ventricular Septal Defect with Eisenmenger Physiology. J Perioper Echocardiogr 2015;3(2):62-65.

\section{Source of support: Nil}

Conflict of interest: None

\section{INTRODUCTION}

Pulmonary hypertension in left-to-right shunt lesions, such as ventricular septal defect (VSD), patent ductus arteriosus, atrial septal defect (ASD) and atrioventricular canal defect, is termed hyperkinetic pulmonary hypertension. ${ }^{1,2}$ It can be the result of an increase in pulmonary blood flow, a direct transmission of the systemic pressure to the pulmonary artery, and an increase in pulmonary vascular resistance (PVR) by compensatory pulmonary vasoconstriction. ${ }^{2}$ The pulmonary vasoconstriction is a defence mechanism for preventing the development of congestive heart failure.

Hyperkinetic pulmonary hypertension is usually reversible if the cause is treated before development of

\footnotetext{
${ }^{1}$ Senior Resident, ${ }^{2}$ Assistant Professor, ${ }^{3}$ Professor and Head

${ }^{1-3}$ Department of Cardiac Anesthesia, All India Institute of Medical Sciences, New Delhi, India

Corresponding Author: Suruchi Hasija, Assistant Professor Department of Cardiac Anesthesia, All India Institute of Medical Sciences, New Delhi, India, Phone: 919868398108 e-mail: suruchi_hasija@hotmail.com
}

permanent changes in pulmonary arterioles. If the large left-to-right shunt lesions are left untreated, irreversible changes take place in the pulmonary vascular bed, leading to severe pulmonary hypertension and cyanosis subsequent to reversal of the left-to-right shunt. This stage is called Eisenmenger's syndrome or pulmonary vascular obstructive disease (PVOD). ${ }^{3}$ Surgical correction is not possible at this stage. The time of onset of PVOD varies, ranging from infancy to adulthood, but the majority of patients develop PVOD during late childhood or early adolescence. $^{4}$

\section{CASE REPORT}

A 12 years old male patient weighing $40 \mathrm{~kg}$ presented with progressively increasing dyspnea on exertion since childhood. There were episodes of cyanosis for last 2 years and chest pain for last 1 year. His electrocardiogram showed left axis deviation and evidence of biventricular hypertrophy. The chest X-ray showed cardiomegaly with signs of pulmonary arterial hypertension. Upon transthoracic echocardiography, patient was diagnosed to have large perimembranous VSD with bidirectional shunt and patent foramen ovale (PFO) with right-to-left shunt, mild pulmonary valve regurgitation and normal biventricle function. Cardiac catheterization showed some pulmonary vascular reactivity to $100 \%$ oxygen. Mean pulmonary artery pressure (PAP) decreased from $70 \mathrm{~mm} \mathrm{Hg}$ on room air to $66 \mathrm{~mm} \mathrm{Hg}$ on oxygen. There was no significant change in PVR.

Patient was scheduled for unidirectional valve patch VSD closure. Patient was given tablet sildenafil $25 \mathrm{mg}$ on the morning of surgery. Anesthesia was induced with opioid-based balanced anesthesia technique. The goals of anesthetic management were to maintain preload, avoid bradycardia, decrease in systemic vascular resistance and increase in PVR, maintain normal sinus rhythm, myocardial contractility and myocardial perfusion. Patient was mechanically ventilated using high concentration of oxygen, $8 \mathrm{ml} / \mathrm{kg}$ tidal volume, respiratory rate sufficient to maintain $\mathrm{PaCO}_{2}$ between 30 and $35 \mathrm{~mm}$ $\mathrm{Hg}$ and $5 \mathrm{~cm} \mathrm{H}_{2} \mathrm{O}$ of PEEP so as to prevent increase in PVR. Intraoperatively, ECG, invasive blood pressure, central venous pressure, pulse oximetry, end-tidal carbon dioxide, bispectral index, urine output, activated clotting 

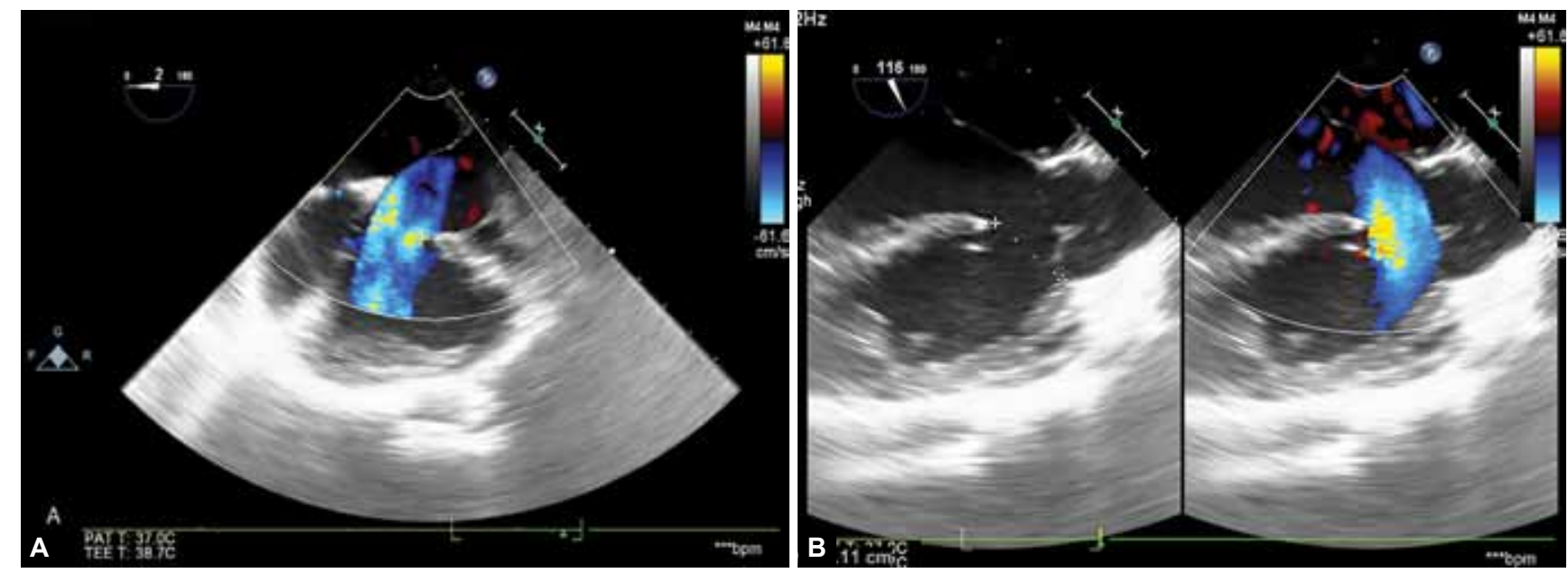

Figs $1 A$ and $B$ : Midesophageal four-chamber view $(A)$ and long-axis view $(B)$ with color flow Doppler showing a large VSD. A PFO can also be seen $(A)$

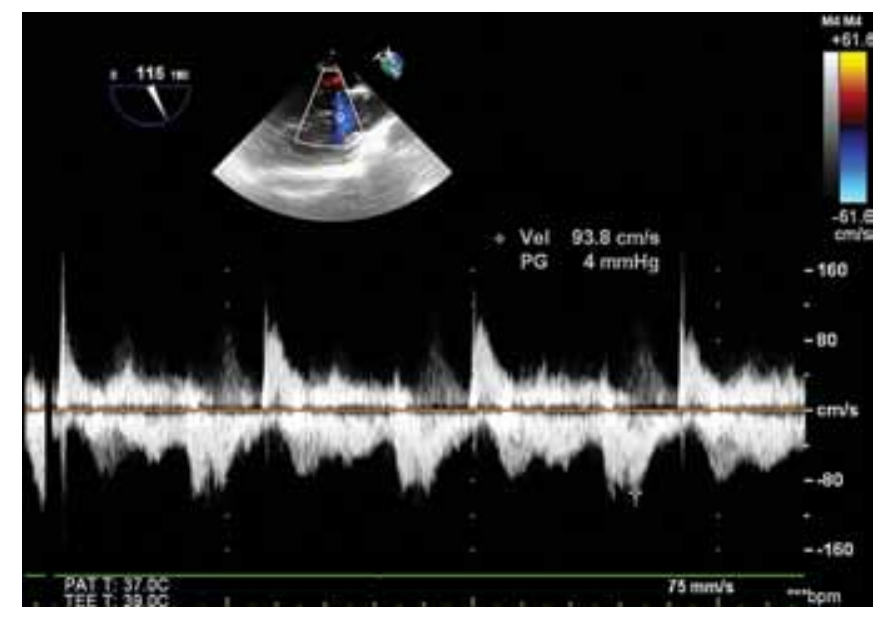

Fig. 2: Midesophageal long-axis view with continuous wave Doppler showing a large unrestrictive VSD with bidirectional flow time, arterial blood gas and acid base status, serum electrolytes, blood sugar, serum lactate and hematocrit were monitored. Transesophageal echocardiography examination (iE33TM; Phillips Medical Systems, Andover, MA) revealed a large perimembranous VSD of size $2 \times 2 \mathrm{~cm}$ with bidirectional shunting, PFO and mild pulmonary regurgitation (Figs 1, 2 and Video 1). The systolic PAP equalled the systolic blood pressure when measured on the operation table by pressure transduction.
A unidirectional valved patch (UVP) VSD closure with polytetrafluoroethylene was performed on cardiopulmonary bypass (CPB) using standard institutional protocol. Phenoxybenzamine $0.5 \mathrm{mg} / \mathrm{kg}$ i.v. was administered slowly after aortic cannulation so as to decrease PVR and facilitate weaning from CPB. The patch had the same width as the size of the defect but approximately one and half times the length of the defect. A $4 \mathrm{~mm}$ fenestration was made into it. The patch was then folded upon itself to achieve adequate dimensions for closure of the VSD covering the fenestration in a valved mechanism (Figs 3A and B). Thereafter, the patch was sutured to the edges of VSD in a way that the flap lay toward the left ventricular side and opened downward toward the left ventricular apex in order to prevent left ventricular outflow tract (LVOT) obstruction during systole. The PFO was closed. The CPB time was 60 minutes and aortic cross clamp time was 40 minutes. There was return of spontaneous sinus rhythm on aortic cross clamp removal. Patient was weaned from CPB with inotropic support of $5 \mu \mathrm{g} / \mathrm{kg} / \mathrm{min}$ dobutamine and $0.5 \mu \mathrm{g} / \mathrm{kg} / \mathrm{min}$ nitroglycerin. Transesophageal echocardiography examination demonstrated the UVP patch with blood flow from right ventricle to left ventricle during systole for a brief period

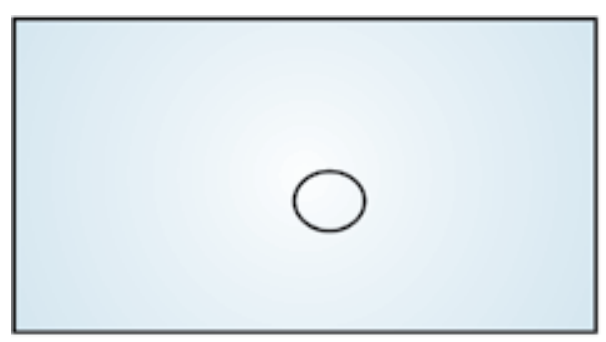

A
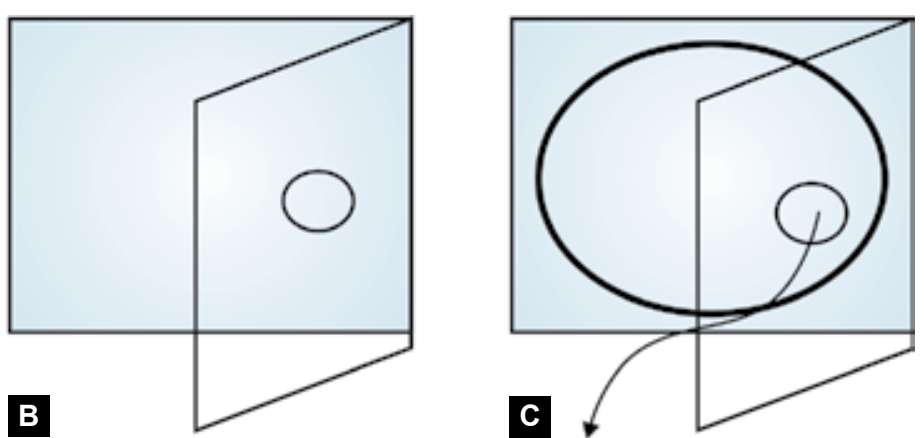

Figs 3A to C: Diagrammatic representation of unidirectional flap valve formation and flow from right to left: (A) VSD patch with fenestration, (B) VSD patch with flap covering the fenestration and (C) VSD patch with flap stitched over the defect 

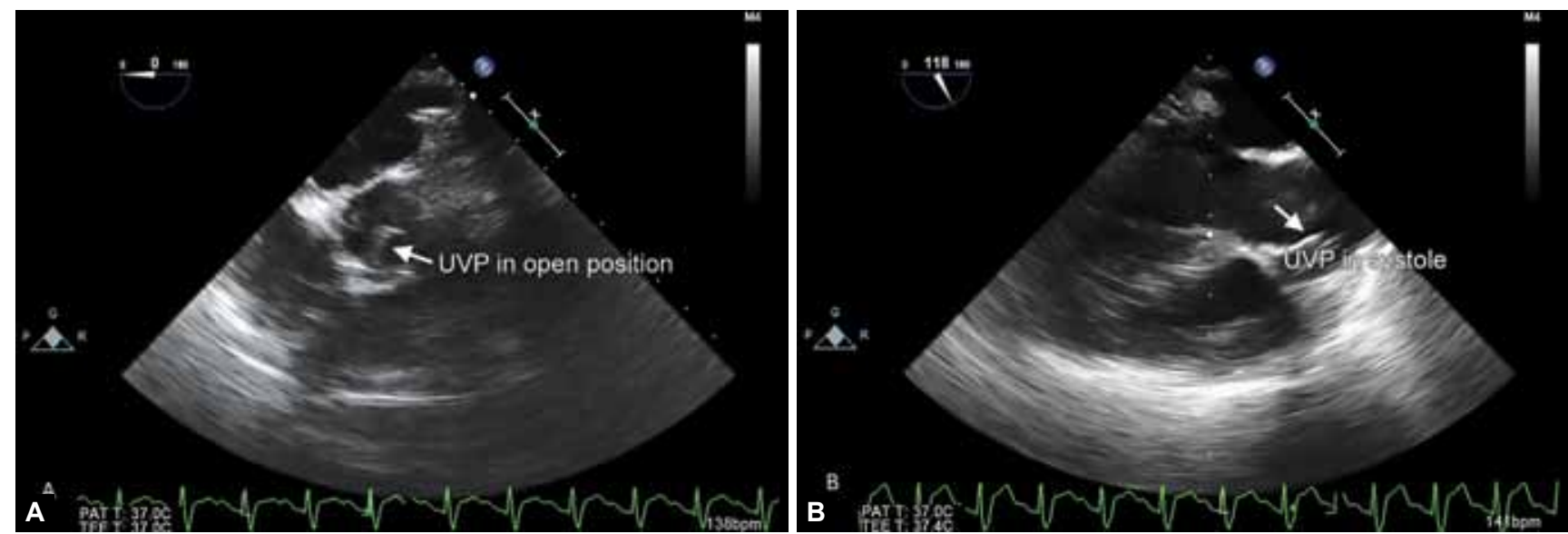

Figs 4A and B: Midesophageal aortic short-axis view (A) and long-axis view (B) showing the unidirectional valved patch (UVP) in open position in systole

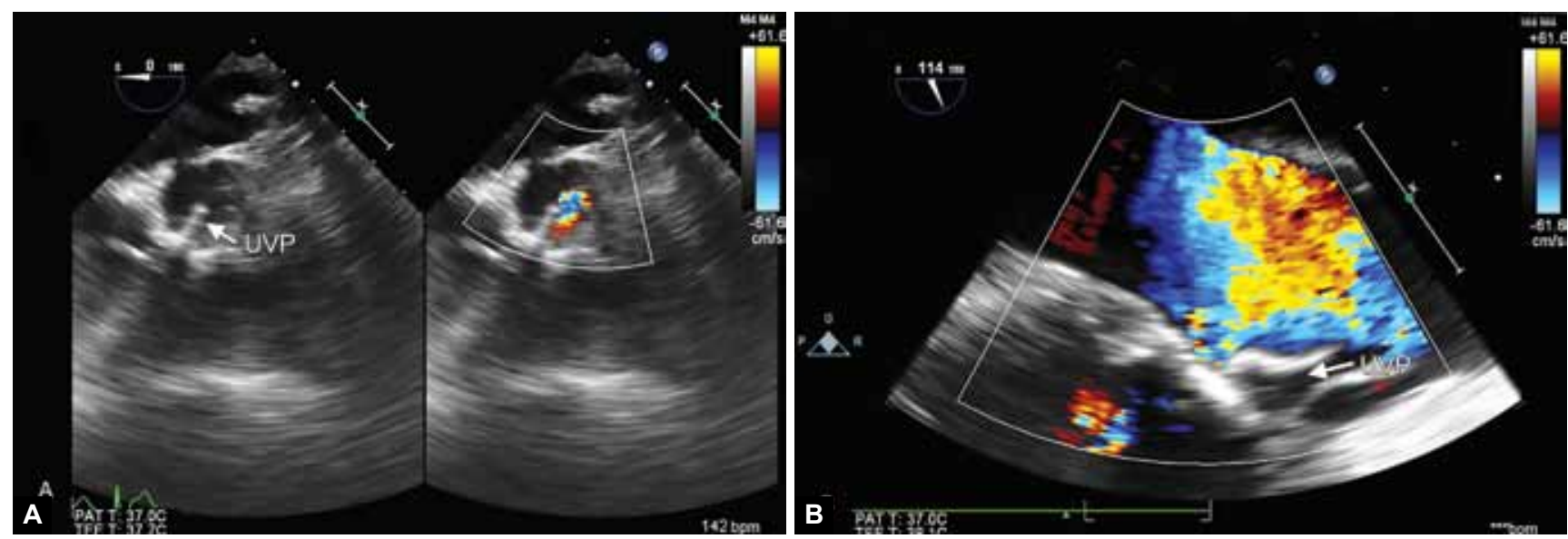

Figs 5A and B: Midesophageal aortic short-axis view (A) and long-axis view (B) with color flow Doppler showing blood flow from right ventricle to left ventricle

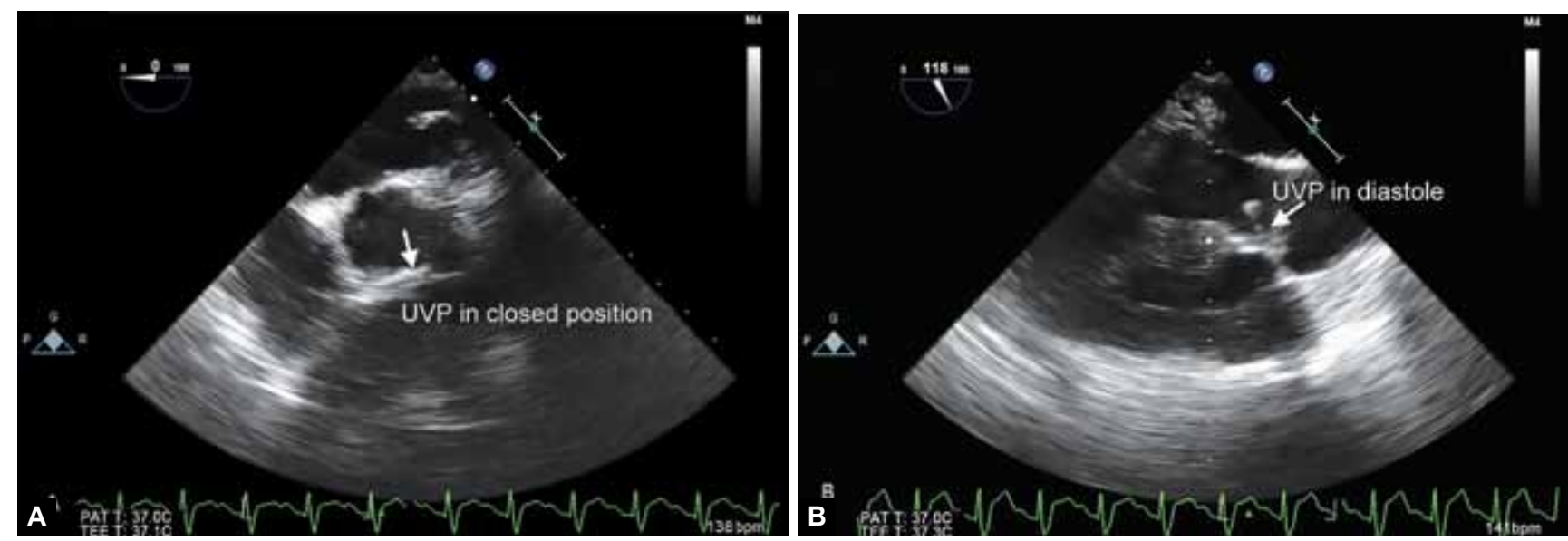

Figs 6A and B: Midesophageal aortic short-axis view (A) and long-axis view (B) showing the unidirectional valved patch (UVP) in closed position in diastole

after weaning from CPB (Figs 4 to 7, Videos 2 and 3), but it ceased after 10 minutes with decrease in PAP.

The patient was shifted to the intensive care unit (ICU) for elective ventilation. He was extubated 6 hours later. He was advised tablet phenoxybenzamine $10 \mathrm{mg}$ TDS and tablet sildenafil $25 \mathrm{mg}$ TDS to lower the PAP in addition to antibiotics, analgesics, diuretic and angiotensin converting enzyme inhibitor. The ICU stay was uneventful. He was shifted to the ward next day and discharged after 5 days.

\section{DISCUSSION}

Elevated pulmonary artery pressure induces varying severity of anatomic changes in the pulmonary vessels. ${ }^{5}$ With sustained pulmonary hypertension, organic changes begin to occur in the pulmonary vasculature 


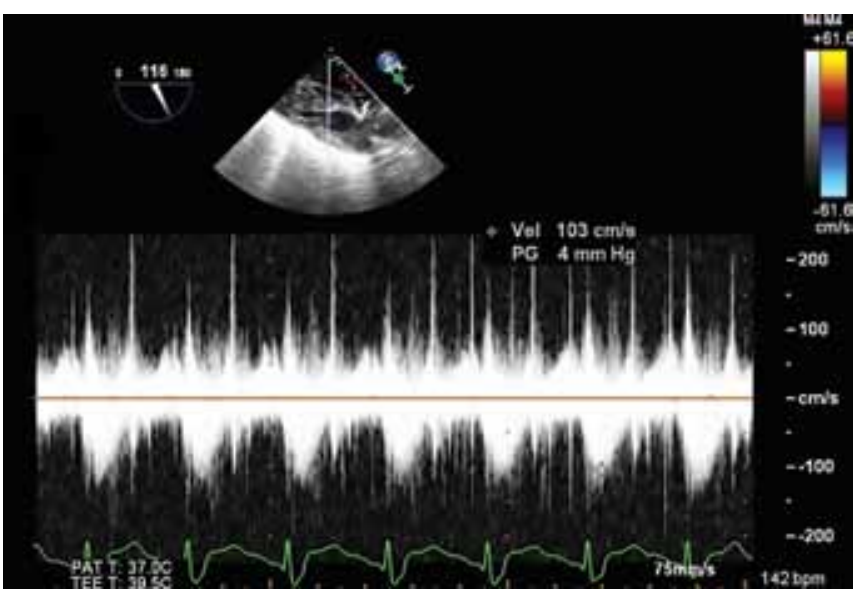

Fig. 7: Transgastric long-axis view with continuous wave Doppler showing laminar flow across the left ventricular outflow tract

which preclude surgical repair. ${ }^{3}$ Previous reports have shown that results after closure of a large VSD with Eisenmenger physiology are dependent upon the severity of pulmonary hypertension at the time of closure. ${ }^{3}$ Patients with VSD and pulmonary hypertension undergoing VSD closure have high mortality and morbidity. In addition, the inflammatory response after CPB leads to release of thromboxane $\mathrm{A} 2$ and catecholamines. These vasoactive substance aggravate pulmonary hypertension. Protamine reaction can also make pulmonary hypertension crisis worse. ${ }^{6}$

Usually, surgeons leave an interatrial communication to decompress the right atrium and right ventricle during pulmonary hypertensive crisis. However, shunting across this communication is often unreliable with the potential for a left-to-right shunt when the pulmonary arterial pressures decrease. ${ }^{7}$

Another approach is a fenestrated VSD patch that converts a large VSD into a small VSD. The fenestration allows for a right-to-left shunt in the case of pulmonary hypertensive crisis. With sustained pulmonary hypertension, the fenestration would allow for a right-to-left shunt with the inherent risk of systemic embolization. But once the PAP decreases, it has the potential for left-to-right shunt along with associated complication of infective endocarditis. $^{8}$

A UVP VSD patch closure is a good alternative in children with pulmonary hypertension and elevated PVR. ${ }^{9}$ It prevents death from acute right ventricular failure and refractory pulmonary hypertension. ${ }^{10}$ For a short period after weaning from $\mathrm{CPB}$, the PAP may be (supra) systemic in patients with severe pulmonary hypertension preoperatively. The UVP at VSD position will shunt from right ventricle to left ventricle for such duration. When the PAP reduces below systemic pressure, the UVP closes to abolish the shunt akin to the PFO. Using color flow and continuous wave Doppler, the direction and magnitude of the shunt can be identified. The systolic PAP in this situation can be estimated as:

Systolic PAP $=$ systolic blood pressure $+\Delta($ RV-LV $)$

Where, $\Delta(\mathrm{RV}-\mathrm{LV})$ is the gradient across the VSD.

Another important consideration is to rule out LVOT obstruction even though the flap is so placed that it opens downward toward the left ventricular during systole and does not impede blood flow.

The technique of UVP VSD closure may allow management of patients with VSD and Eisenmenger physiology at risk of pulmonary hypertensive crises postoperatively in the absence of facilities of extracorporeal membrane oxygenation and nitric oxide ventilation.

\section{REFERENCES}

1. Widlitz A, Barst JR. Pulmonary arterial hypertension in children. Eur Respir J 2003;21(1):155-176.

2. Park KM. Pediatric cardiology for practitioners. 5th ed; Philadelphia: Elsevier; 2008. p. 593.

3. Rabinovitch M, Keane JF, Norwood WI, Castaneda AR, Reid L. Vascular structure in lung tissue obtained at biopsy correlated with pulmonary hemodynamic findings after repair of congenital heart defects. Circulation 1984;69(4):655-667.

4. Blackstone EH, Kirklin JW, Bradley EL, DuShane JW, Appelbaum A. Optimal age and results in repair of large ventricular septal defects. J Thorac Cardiovasc Surg 1976;72(5): 661-667.

5. Forfia PR. Approach to patients with heart failure and pulmonary hypertension. Curr Treat Options Cardiovasc Med 2007;9(4):302-309.

6. Hopkins RA, Bull C, Haworth SG, et al. Pulmonary hypertensive crises following surgery for congenital heart defects in young children. Eur J Cardiothorac Surg 1991; 5(12):628-634.

7. Collins TJ, Moore JW, Kirby WC. Atrial septostomy for pulmonary hypertension. Am Heart J 1988;116(3):873-874.

8. Novick WM, Gurbuz AT, Watson DC, et al. Double patch closure of ventricular septal defect with increased pulmonary vascular resistance. Ann Thorac Surg 1998;66(5):1533-1538.

9. Talwar S, Choudhary SK, Airan B, Saxena A, Kothari SS, Juneja R. Unidirectional valved patch for closure of septal defects in patients with severe pulmonary hypertension. Ann Pediatr Cardiol 2008;1(2):114-119.

10. Choudhary SK, Talwar S, Airan B. A simple technique of unidirectional valved patch for closure of septal defects. J Thorac Cardiovasc Surg 2007;134(5):1357-1358. 